\title{
Fabrication of Electrodes for Chemical Sensors on Overhead- Transparency Film
}

\author{
Shigeru Toyama, ${ }^{\text {* }}$ Kazuhito Aoki, ${ }^{\text {a }}$ Seishi Kato, ${ }^{\text {a }}$ \\ Masanori NAKAMURA, ${ }^{\mathrm{b}}$ and Ron USAMI ${ }^{\mathrm{b}}$

\begin{abstract}
aResearch Institute, National Rehabilitation Center for Persons with Disabilities (4-1 Namiki, Tokorozawa, Saitama 359-8555, Japan)

${ }^{b}$ Department of Applied Chemistry, Faculty of Engineering, Toyo University (2100 Nakanodai, Kujirai, Kawagoe,
\end{abstract} \\ Saitama 350-0815, Japan)
}

Received August 26, 2005 ; Accepted November 4, 2005

\begin{abstract}
Metal electrodes for chemical sensors have been fabricated on an overhead-transparency film using the Line Patterning Method. This method comprises i) laser-printing of toner with negative pattern of the electrodes, ii) vapor-deposition of metal layers, and iii) removal of the toner and the upper metal layers. The resolution of the pattern was $c a .0 .1-0.2 \mathrm{~mm}$. We found that multilayered electrodes such as $\mathrm{Au} / \mathrm{Cr} / \mathrm{film}, \mathrm{Pt} / \mathrm{Au} / \mathrm{Cr} / \mathrm{film}$, and $\mathrm{Ag} / \mathrm{Pt} / \mathrm{Au} / \mathrm{Cr} /$ film satisfied conductivity, adhesiveness, and flexibility. Furthermore, a glucose sensor using the film electrode was fabricated.
\end{abstract}

Key Words : Overhead-Transparency Film, Metal Electrodes, Chemical Sensor, Glucose Sensor

\section{Introduction}

Disposable chemical sensors are now indispensable tools for monitoring the health of the individuals. In the case of electrode-based disposable sensors, screen-printing is a widely used fabrication method whereby simple, fast, and cost beneficial production is possible. However, it requires a patterned screen often requires special equipments. Therefore, it is inconvenient to use it in the development stage, especially in laboratory scale. Here, we describe electrodes that are suitable for fabricating disposable chemical sensors using the "Line Patterning Method".

The Line Patterning Method, a kind of lift-off method originally invented by the group of MacDiarmid, ${ }^{1-3)}$ is a method to prepare a patterned layer of conducting polymer $^{2-5)}$ on an overhead-transparency film. The patterning process of this method is based on the laser printing, and a polymer pattern is obtained by removing the polymer together with the underlying toner from the film surface that is coated by the polymer in advance. The crucial point of this method is that the printer toner dissolves in an organic solvent but the conductive polymer does not.

Here, we fabricated various metal electrodes on an overhead-transparency film by e-beam vapor deposition. In the case of chemical sensors, noble metal electrodes such as $\mathrm{Pt}, \mathrm{Ag}$, and Au play important roles. For example, $\mathrm{Pt}$ is used as a counter electrode, and $\mathrm{Ag}$ as a reference electrode. Au is often used for biosensors because it can tightly immobilize biomaterials via a mercaptide group. The purpose of this article is to show the fabrication process of these electrodes on an overhead-transparency film. We customized the Line Patterning Method for this purpose. To show the usefulness of the fabrication process of such electrodes, we further fabri- cated a glucose sensor as an application example.

\section{Experimental}

The overhead-transparency film (KOKUYO VF-1, poly(ethylenetelephthalate), $297 \times 210 \mathrm{~mm}^{2}$, thickness $=$ $100 \mu \mathrm{m})$ was used as the substrate. An electrode pattern was negatively printed on the film with a laser printer (EPSON LP-8700PS3). The film was then transferred into the chamber of an electron beam evaporator (ULVAC EBX-8C), and metals were evaporated. After the formation of metal layers, the film was cut into individual chips, which was dipped into acetone and sonicated for several minutes to remove toner and upper metal layers.

The structure of the glucose sensor is shown in Fig. 1. It consists of three electrodes: an enzyme-immobilized electrode, an $\mathrm{Ag} / \mathrm{AgCl}$ electrode, and a counter electrode. The basal electrode for the glucose sensor was prepared by following the above method. In this case, $\mathrm{Cr}$ (5 nm), Au (40 nm), Pt (30 nm), and Ag (200 nm) were sequentially deposited in this order. We could prepare 70 basal electrodes from an A4-sized overhead-transparency film at a time. Then the surface Ag layer of two electrodes was selectively removed by applying $1000 \mathrm{mV}$ (vs. $\mathrm{Ag} / \mathrm{AgCl}$ ) in $18 \% \mathrm{HNO}_{3}$ solution for 5 sec. The sur-

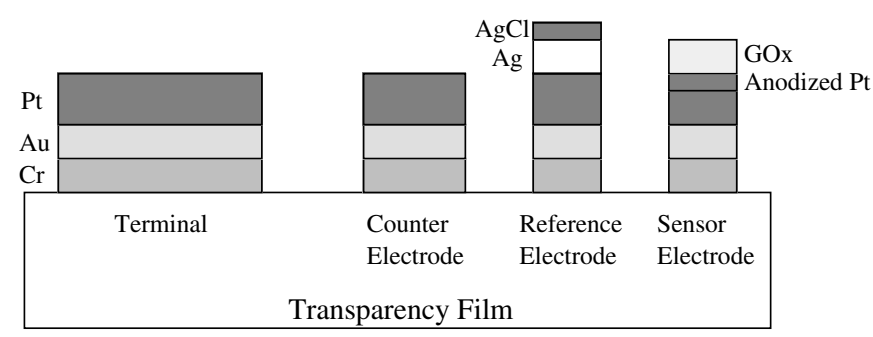

Fig. 1 Cross-sectional structure of the glucose sensor. 
face of the remaining silver electrode was oxidized to form $\mathrm{AgCl}$ by applying constant current $(100 \mu \mathrm{A})$ for 10 $\mathrm{sec}$ in a $100 \mathrm{mM}$ phosphate buffer solution (pH 6.9) containing $200 \mathrm{mM} \mathrm{NaCl}$. The sensor was accomplished by immobilizing glucose oxidase (GOx, from Aspergillus sp., TOYOBO ENZYMES) on the working electrode of the chip. Before the immobilization step, the surface of the working electrode $(\mathrm{Pt})$ was made inert to suppress nonspecific response to reducing sugars by applying 1200 $\mathrm{mV}$ (vs. $\mathrm{Ag} / \mathrm{AgCl}$ ) for $15 \mathrm{~min}$ in a $100 \mathrm{mM}$ phosphate buffer solution (pH 6.9). ${ }^{6}$ ) Then $5 \mu$ l of a GOx solution $(1.23 \mu \mathrm{U} / \mu \mathrm{l})$ was dropped onto the sensor electrode and incubated for $30 \mathrm{~min}$ to adsorb GOx on the electrode surface. The remaining GOx was rinsed away, and then a $1 \%$ glutaraldehyde solution $(5 \mu \mathrm{l})$ was dropped on the electrode and incubated for $30 \mathrm{~min}$. After rinsing to remove glutaraldehyde, the sensor chip was dried. Finally, another overhead-transparency film as an insulator was attached on the chip with a double-sided adhesive tape.

\section{Results and Discussion}

Various metals such as $\mathrm{Cr}, \mathrm{Ag}, \mathrm{Au}, \mathrm{Ti}$, and Pt were successfully deposited on the film by e-beam vacuum evaporation. The overhead-transparency film was not damaged by exposure to the heat source during evaporation. This is one of the advantages of using the overhead-transparency film. We also successfully deposited Al on the film by RF sputtering in the preliminary experiments. However, it was hard to obtain a combination of good conductivity, adhesiveness, and flexibility in the case of a film using only single metal layer.

The conductivity of Au and Ag layers was satisfactory while that of $\mathrm{Cr}$, Ti, and Pt layers was not as good in terms of comparison with bulk conductivity.

Through the evaluation of a tape-peeling test, we found that the adhesiveness of $\mathrm{Cr}, \mathrm{Ti}$, and $\mathrm{Pt}$ on the film was excellent while that of $\mathrm{Au}$ and $\mathrm{Ag}$ was moderate. We further tested the adhesiveness after the incubation of metal-deposited films in an autoclave $\left(121^{\circ} \mathrm{C}, 60 \mathrm{~min}\right)$. The adhesiveness of $\mathrm{Cr}$, Ti, and $\mathrm{Pt}$ was not affected by this treatment, meanwhile that of $\mathrm{Au}$ and $\mathrm{Ag}$ was seriously decreased.

To maintain flexibility, which is one of the advantageous properties of the overhead-transparency film, the electrodes must be durable against folding. However, the conductivity of $\mathrm{Cr}$-, Ti-, and Pt-deposited films decreased by folding, while that of Au- and Ag-deposited films was not seriously changed. Figure 2 shows SEM photographs of the surface of Ti- and Au-deposited overhead-transparency films after repeated foldings. Mutually isolated islands were observed in a Ti layer, while many but short cracks were observed in an Au layer. The decrease in the conductivity of the Ti-deposited film after the folding is probably due to the formation of many electrically isolated domains.

Adequate conductivity, adhesiveness, and flexibility were achieved with multilayered metal films. For instance, inserting $\mathrm{Cr}$ between $\mathrm{Au}$ and the transparent film improved the adhesiveness of Au without sacrificing conductivity and flexibility. We also discovered that $\mathrm{Pt} / \mathrm{Au} / \mathrm{Cr} / \mathrm{film}, \mathrm{Ag} / \mathrm{Pt} / \mathrm{Au} / \mathrm{Cr} / \mathrm{film}$, and $\mathrm{Ag} / \mathrm{Au} / \mathrm{Cr} / \mathrm{film}$ satisfied all these properties. Currently, we do not know why the insertion of $\mathrm{Cr}$ improved the adhesiveness between $\mathrm{Au}$ and the film.

The resolution of the patterning method was also evaluated. Figure 3 shows the positive and negative lines of $\mathrm{Ag}$ directly deposited on an overhead-transparency film. In the case of negative lines, islands were observed in

a)

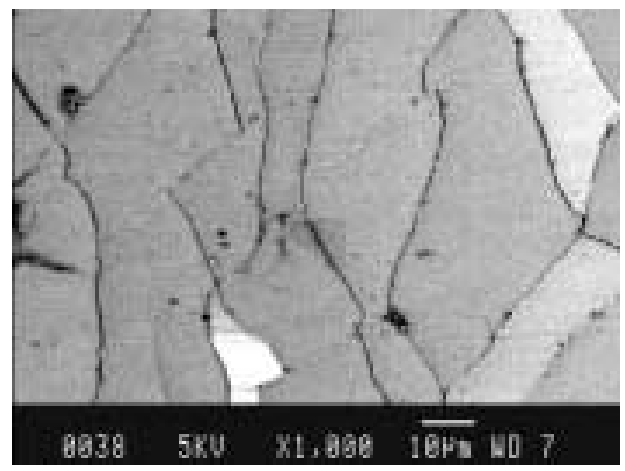

b)

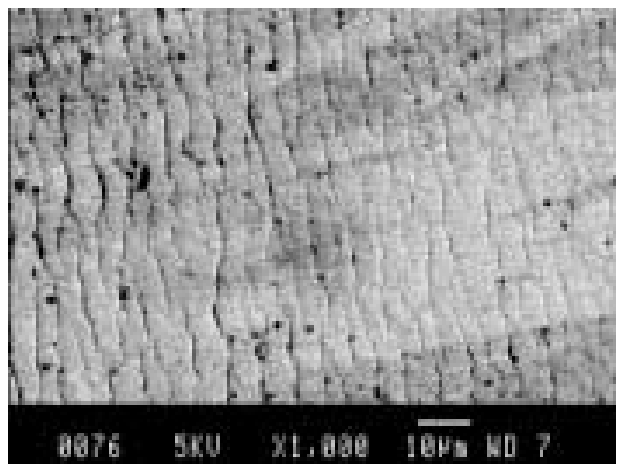

Fig. 2 SEM photographs of the surface of overheadtransparency film deposited with a) Ti (400 nm) or b) Au $(120 \mathrm{~nm})$. Photographs were taken after repeated foldings (5 times).
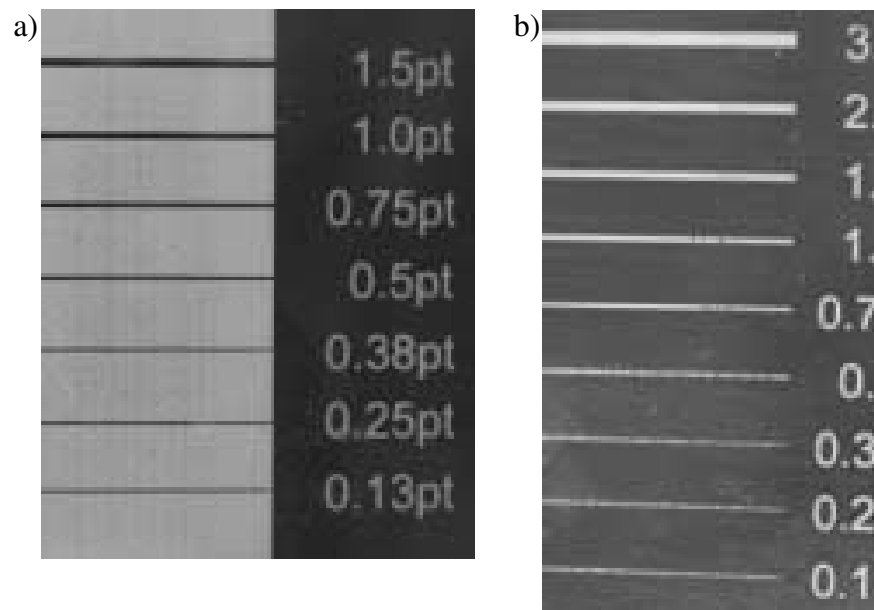

Fig. 3 Line patterns of Ag on overhead-transparency films (a: positively patterned lines, b: negatively patterned lines). In the photographs, black and white areas are corresponding to the Ag-coated and -noncoated parts, respectively. 


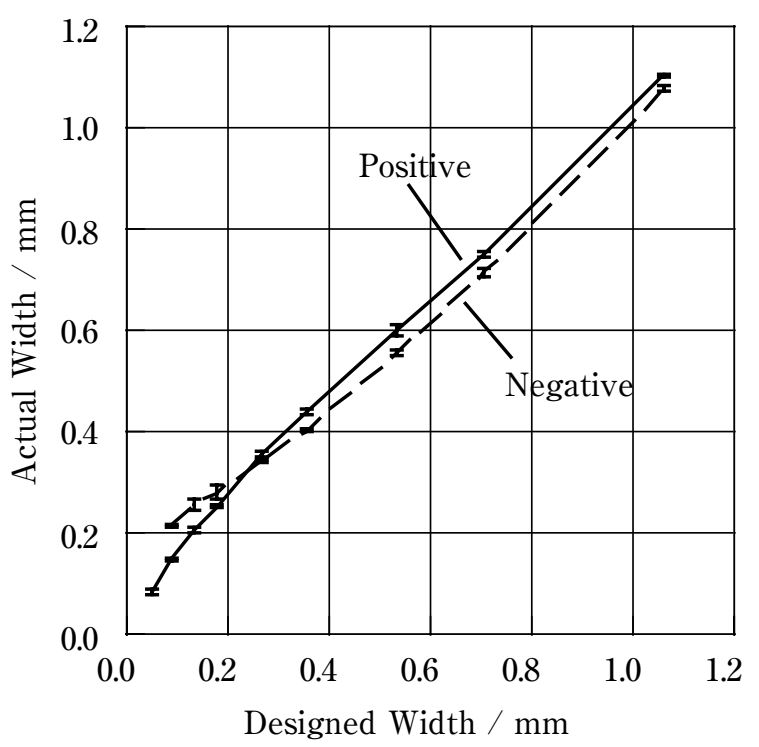

Fig. 4 Widths of positive and negative line patterns obtained by the length measurement using microscope $(n=5)$ versus designed line width.

the lines when the line width became narrow. Such islands, which seems to originate from the toner pattern on the overhead-transparency film, limited the resolution of the patterning method. The printing resolution on overhead-transparency films is less than that on papers, and this tendency is more or less common in the case of using other laser printers. The resolution of the patterning method was further evaluated by measuring the line thickness. Figure 4 shows the printed width versus the designed width of line patterns, giving us important suggestions for pattern designing. Relatively large discrepancy between the widths of actual and designed lines was observed below $0.5 \mathrm{~mm}$, especially in the negative line. The practical resolution of the pattern was ca. 0.1$0.2 \mathrm{~mm}$.

We further demonstrated a glucose sensor (Fig. 5) in which Ag and Pt electrodes were fabricated on the same chip. We could also fabricate $\mathrm{Ag}$ and Au electrodes on the same chip. Figure 6 shows a typical response curve of the glucose sensor. The current of the sensor increased step by step with the addition of a glucose solution. The addition of fructose induced negligible response, suggesting that the sensor was specific for glucose. Thus, we could successfully fabricate a chemical sensor on an overhead-transparency film.

\section{Conclusion}

This work provides a simple electrode fabrication method for chemical sensors. We found best combination of metal layers that satisfied conductivity, adhesiveness, and flexibility. We also provided a method to fabricate $\mathrm{Pt}$ and $\mathrm{Ag}$ electrodes on a single film. Furthermore, we fabricated a glucose sensor based on the film electrode as an application example of this method.

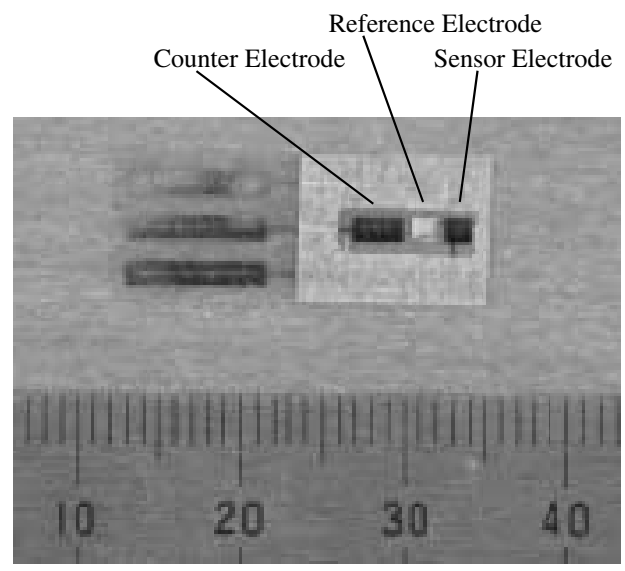

Fig. 5 Photograph of the glucose sensor.

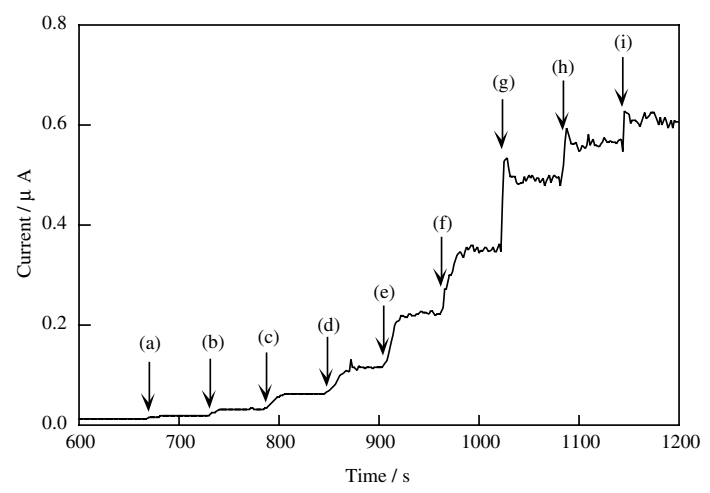

Fig. 6 Time course of the response of the glucose sensor. The sensor chip was immersed into a phosphate buffer solution (100 mM, pH 6.9) containing $200 \mathrm{mM} \mathrm{NaCl}$, and 700 $\mathrm{mV}$ was applied on the sensor electrode vs. a reference electrode. Eventually, concentrated sugar solutions (1 M fructose (a) or glucose (b-i) buffered with $100 \mathrm{mM}$ phosphate, also containing $200 \mathrm{mM} \mathrm{NaCl}$ ) were repeatedly added into the solution at the time indicated by arrows. The final concentrations from (a) to (i) are 2.0 (fructose), 0.5, 1.5, 3.5, 8.4, 18, 37, 55, $73 \mathrm{mM}$ (glucose), respectively.

\section{Acknowledgement}

We would like to thank Dr. Okuzaki in Yamanashi Univ. for kindly demonstrating the original Line Patterning process.

\section{References}

1) A. G. MacDiarmid, D. Hohnholz, and H. Okuzaki, PCT/US01/15773 (2001).

2) D. Hohnholz and A. G. MacDiarmid, Synthetic Metals, 121, 1327 (2001).

3) D. Hohnholz, H. Okuzaki, and A. G. MacDiarmid, Adv. Funct. Mater., 15, 51 (2005).

4) H. Okuzaki, M. Ishihara, and S. Ashizawa, Synthetic Metals, 137, 947 (2003).

5) A. Michalska, M. Ocypa, and K. Maksymiuk, Electroanalysis, 17, 327 (2005).

6) Y. Ikariyama, N. Shimada, S. Yamauchi, T. Yukiashi, and H. Ushioda, Anal. Lett., 21, 953 (1988). 